\title{
Comparative Evaluation of Leukotoxic Activities of Indigenous Staphylococcus aureus Isolates from Subclinical and Clinical Mastitic Milk Samples of Buffalo and Cattle
}

\author{
Jamal Muhammad Khan ${ }^{1}$, Muhammad Hidayat Rasool ${ }^{*}, 2$, Muhammad Arshad ${ }^{1}$, Sajjad Ur Rahman ${ }^{1}$, \\ Muhammad Farooq Tahir ${ }^{1}$, Bilal Aslam², Wen Jing ${ }^{3}$, Zou Jun ${ }^{3}$ and Moeez Ghani ${ }^{4}$ \\ ${ }^{1}$ Institute of Microbiology, University of Agriculture Faisalabad, Pakistan \\ ${ }^{2}$ Department of Microbiology, Govt. College University Faisalabad, Pakistan \\ ${ }^{3}$ National Laboratory of Vet. Parasitology, China Agricultural University, Beijing \\ ${ }^{4}$ Institute de Recherche en Biothérapie (IRB), France
}

\begin{abstract}
The present study was conducted for the comparative evaluation of leukotoxic activities of indigenous Staphylococcus aureus isolates issued from Institute of Microbiology University of Agriculture Faisalabad Pakistan. A total of 200 milk samples 100 each of buffalos (Nili Ravi) and cattle (Sahiwal) with subclinical and clinical mastitis were collected. Staphylococcus aureus isolates recovered were used for extraction of leukotoxins. Leukotoxic activities were determined against bovine Polymorphonuclear cells (PMNs) through Toxin Limit Concentration (TLC) assay. Overall positive percentage of mastitis was more important in cattle (32\%) than for buffalos $(22 \%)$. Overall and quarter based prevalence of subclinical mastitis were higher than clinical cases in both buffalos and cattle. In TLC assay, PMNs remained normal in control wells without leukotoxins after incubation at $37^{\circ} \mathrm{C}$ for 30 minutes, whereas, under the influence of leukotoxins, PMNs flattened and spread on the gelatin coated plastic surface. TLC titers in subclinical mastitis ranged from 4 to 16 with Geometric Mean Titer (GMT) at 6 in buffalos and 8 in cattle whereas, in clinical cases it ranged from 32 to 128 with GMT of 64 in buffalo and cattle.
\end{abstract}

Keywords: Staphylococcus aureus, mastitis, leukotoxins, toxin limit concentration.

\section{INTRODUCTION}

Staphylococcus aureus is the most prevalent and economically important mammary pathogen. It is responsible for 30-40 percent of mastitis cases in dairy animals $[1,2]$. Staphylococcal infections reduce milk yield and exert deleterious effects on milk composition one of which is increase in milk somatic cells [3]. The entry of $S$. aureus into the mammary gland is attributed to the contamination from the environment and from cow to cow mainly during milking [4]. S. aureus produces several virulence factors including surface-associated products and secreted proteins like leukotoxins and enterotoxins $[5,6]$.

The staphylococcal leukotoxins constitute a family of pore-forming binary toxins acting in two steps, first by the binding of the class $\mathrm{S}$ component at the surface of the cellular membrane and then by the association of the class $\mathrm{F}$ component to the complex membrane-class S component [7]. Moreover LukM is a major agent of the leukotoxicity of positive mastitis isolates [7]. To reduce mastitis frequency, current methods rely heavily on therapeutic and prophylactic

*Address correspondence to this author at the Department of Microbiology, Govt. College University Faisalabad, Faisalabad, 38000, Pakistan; Tel: +92301-7102378, +92-41-9201205;

E-mails:drmhrasooluaf@hotmail.com,drmhrasooluaf@gmail.com measures, but the cure rate for treatment with antibiotics is not up to the mark for mastitis pathogen such as Staphylococcus aureus, which accounts for $>30 \%$ of clinical cases. Polymorphonuclear cells (PMNs) indeed form the first line of immunity and cellular defense against mastitic pathogens. To cause mastitis, $S$. aureus must gain access to the mammary gland through the teat canal and overcome local humoral and cellular immune defenses [8].

The leukotoxins produced by $S$. aureus act as virulence factors and contribute to the pathogenesis of mastitis and evasion of the immune system. Leukotoxins target leucocytes of myeloid origin, in particular granulocytes and monocytes. S. aureus strains producing leukotoxins may interfere with host PMNs defense mechanisms as these toxins target macrophages, monocytes and PMNs. The invivo production of these leukotoxins was confirmed by increased antibody titers in milk and serum of experimentally infected goats [9].

Keeping in view the importance of the subject, the present study was conducted for the comparative evaluation of leukotoxic activities of indigenous isolates of $S$. aureus recovered from subclinical and clinical mastitic milk samples of buffalo and cattle against bovine PMNs using Toxin Limit Concentration (TLC) assay. 


\section{MATERIALS AND METHODS}

\subsection{Collection of Milk Samples}

A total of two hundred lactating buffalo and cows (100 each) were selected randomly for the collection of milk samples. All information regarding the health status of animals and previous infection was also recorded. Milk samples were collected after sterilization of teats. Surf Field Mastitis Test (SFMT) was carried out for detection of mastitis according to the method described by Muhammad [10]. About $10 \mathrm{ml}$ of milk was collected in sterile test tubes from positive quarters in the middle of collection.

\subsection{Isolation and Identification of Staphylococcus aureus}

The collected milk samples were inoculated on Staph. $110^{\circledR}$ agar medium (Oxoid UK) and. incubated at $37^{\circ} \mathrm{C}$ for 24-48 hours for the isolation of Staphylococcus aureus. The cultural \& colony characteristics were recorded and Gram's staining was performed for the microscopic identification through shape, arrangement and staining character. Blood agar (Difco USA) was used for the evaluation of hemolytic properties of $S$. aureus and biochemical tests including catalase \& coagulase tests were performed for the confirmation of isolates.

\subsection{Production and Extraction of Leukotoxins}

The indigenous $S$. aureus isolates were cultured in Brain Heart Infusion (BHI) broth (Difco USA) overnight at $37^{\circ} \mathrm{C}$ with vigorous shaking at $150 \mathrm{rpm}$ to promote luxuriant production of toxins. The test tubes containing BHI broth with bacterial growth were centrifuged at $9000 \times \mathrm{g}$ for 30 min and culture supernatants suspected to contain $S$. aureus leukotoxins were filtered using $0.45 \mu \mathrm{m}$ pore size filters. The filtered leukotoxins were stored at $-20^{\circ} \mathrm{C}$ for further use [7].

\subsection{Isolation of Bovine Polymorphonuclear Cells (PMNs)}

The isolation of Polymorphonuclear cells (PMNs) was carried out according to the method described by Carlson and Kaneko [11]. A $10 \mathrm{ml}$ bovine blood was collected in sterile test tube having EDTA (Vacutainer ${ }^{\mathrm{TM}}$ ). The selected animal for the collection of blood was treated with antiparasitic drug (Ivermectin $6 \mathrm{ml}$ ) to reduce the number of eosinophils in blood. The anticoagulant added blood was centrifuged at $8000 \times \mathrm{g}$ for $10 \mathrm{~min}$ at room temperature. The WBCs collected from buffy coat containing residual RBCs were suspended in hypotonic solution in 3:1 ratio and gently rotated for the lysis of RBCs. Suspension was centrifuged at $100 \times g$ for 5 min and pellet containing cells was washed using Hank's Balanced Salt Solution (HBSS) and supernatant was discarded. The pellet was finally suspended in $5 \mathrm{ml}$ of RPMI-1640 medium without sodium carbonate supplemented with $0.1 \%(\mathrm{w} / \mathrm{v})$ gelatine (Difco USA) and $20 \mathrm{mM}$ HEPES. The viability of cells was observed by trypan blue dye and final volume of $2 \times 10^{6} \mathrm{cells} / \mathrm{ml}$ was adjusted [7].

\subsection{Toxin Limit Concentration (TLC) Assay}

The wells of 96-well flat-bottom microtiteration plate (Titertek UK) were coated with $0.5 \%$ gelatine (Difco USA) in water for 15 minutes at $37^{\circ} \mathrm{C}$. Serial two fold dilutions of crude leukotoxins in PBS were distributed in all the wells along with a control (culture medium without toxins). To every well, $20 \mu 1$ of PMNs suspension was added and the plate was incubated at $37^{0} \mathrm{C}$ for $30 \mathrm{~min}$. The shape of PMNs was then examined under an inverted microscope. The last dilution inducing the spreading of more than $95 \%$ PMNs was taken as the end point and the TLC titer was the inverse of this dilution [7]. The Geometric Mean Titer (GMT) of TLC titers of leukotoxins isolated from buffalos and cattle in subclinical and clinical cases of mastitis were determined.

\section{RESULTS}

Milk samples were collected randomly from buffalo and cattle of different ages and stages of lactation from in and around Faisalabad Pakistan. The Surf Field Mastitis (SFM) test was applied for the detection of subclinical and clinical mastitis. Out of 100 cattle examined, the overall positive percentage of mastitis was $32 \%$ with $17 \%$ cases of subclinical and $15 \%$ of clinical mastitis. Similarly, out of 100 buffalo examined, the overall positive percentage of mastitis was $22 \%$ with $13 \%$ cases of subclinical and $9 \%$ of clinical mastitis. A total of 400 quarters were examined in each of buffalo and cattle separately. The quarter based prevalence of mastitis was recorded same $(12.5 \%)$ in buffalo as well as in cattle. The prevalence of quarter based subclinical and clinical mastitis in cattle was found to be $8 \%$ and $4.5 \%$, respectively. Similarly, in buffalo quarter based prevalence of mastitis in subclinical and clinical conditions was $8.5 \%$ and $4 \%$, respectively.

The mastitic milk samples from buffalo and cattle were processed further for the isolation of $S$. aureus. After inoculation of 50 positive milk samples each from buffalo and cattle on Staph 110 agar medium, $45(90 \%)$ samples in case of cattle and $42(84 \%)$ in buffalo showed growth. Smooth, circular, raised and golden yellow colonies of Staphylococci with 1-2 mm diameter were observerd. On Gram's staining, G+ve cocci with grapes like appearance were found. All the isolates on Staph 110 agar medium showed haemolytic properties with pin head, convex colonies of 1-2 mm diameter when inoculated on to the blood agar plates. The positive results of catalase and coagulase tests confirmed the presence of $S$. aureus.

For the production of leukotoxins from indigenous isolates, Brain Heart Infusion (BHI) broth was used. Leukotoxins were extracted in crude form 8 isolates of subclinical and 15 isolates of clinical mastitis. Polymorphonuclear cells (PMNs) were isolated from bovine blood and viability tested using trypan blue dye. The cell suspension with more than $95 \%$ viability were maintained at $2 \times 10^{6} \mathrm{cells} / \mathrm{ml}$ and used in Toxin Limit Concentration (TLC) assay.

To determine the toxic effects of crude leukotoxins on bovine PMNs, the TLC assay was performed. After 30 minutes of incubation at $37^{\circ} \mathrm{C}$, the shape of PMNs was examined with an inverted microscope. Under the influence of leukotoxins, PMNs flattened and spread on the gelatin coated plastic surface whereas in control well without toxins, the cells remained normal as shown in Figs. $(\mathbf{1}, \mathbf{2})$. The TLC titers of leukotoxins extracted out of $S$. aureus isolates recovered from subclinical mastitic cases of buffalo and cattle ranged from 4 to 16 with GMT of 6 in buffalo and 8 in cattle, whereas, the TLC titers of clinical mastitic cases of buffalo and cattle ranged from 32 to 128 with same GMT of 
64. The comparative GMT of buffalo and cattle in clinical cases of mastitis are shown in Fig. (3).

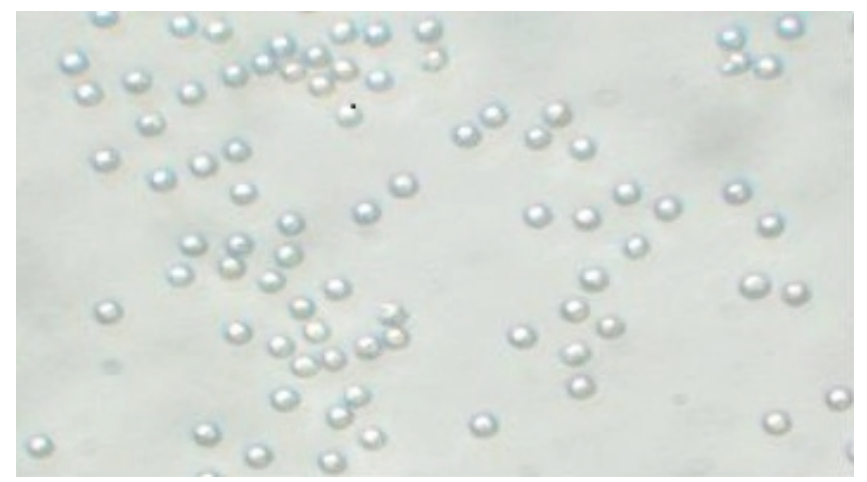

Fig. (1). The intact monolayer of bovine PMNs after 30 minutes of incubation at $37^{\circ} \mathrm{C}$ in the absence of leukotoxins (200X).

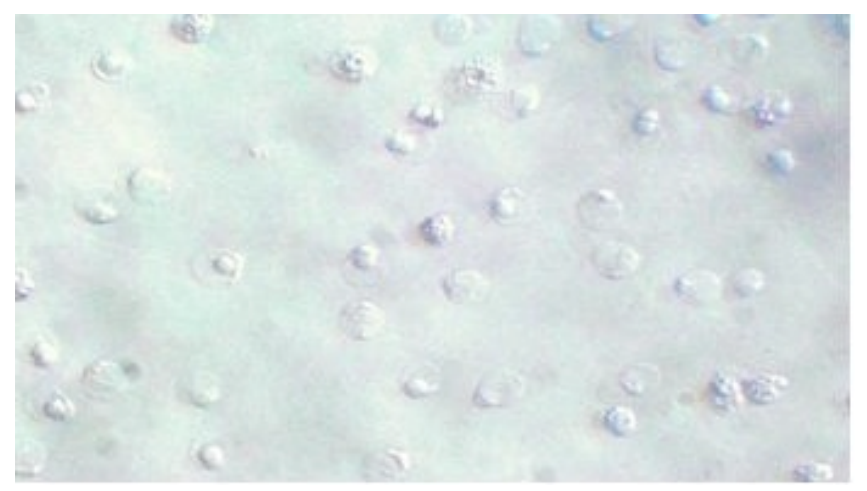

Fig. (2). The spreading of bovine PMNs after 30 minutes of incubation at $37^{\circ} \mathrm{C}$ with crude leukotoxins (200X).

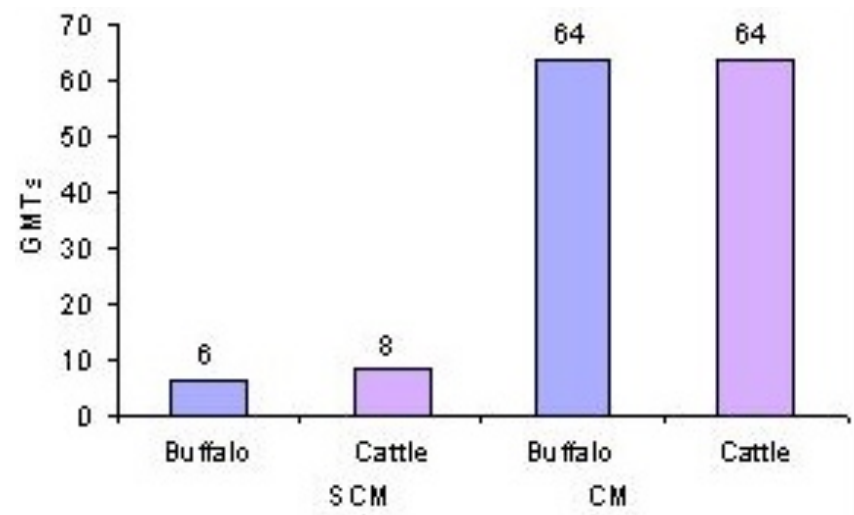

Fig. (3). Comparative GMTs of Toxin Limit Concentration (TLC) titers of leukotoxins extracted from Subclinical Mastitis (SCM) and Clinical Mastitis (CM) cases of buffalo and cattle.

\section{DISCUSSION}

Mastitis is the most prevalent and economically important disease of dairy animals throughout the World. This disease complex is the out-come of interaction of multiple factors. These factors are associated with the host, the pathogen and the environment. Among the pathogens, Staphylococcus aureus is the most common cause of bovine mastitis and is responsible for the main economic loss to dairy industry $[12,13]$. Bicomponent leukotoxins are known to be secreted by some strains of mastitis causing $S$. aureus but information on the prevalence of leukotoxins secreted by indigenous isolates and their level of toxicity in subclinical and clinical cases of mastitis are not available in Pakistan. This prompted us to conduct the present study for the first time in the country to gain some information on the comparative leukotoxic activity of indigenous $S$. aureus isolates.

Out of 100 collected milk samples each from buffalo and cattle, the overall prevalence of mastitis was found to be higher in cattle (32\%) than buffalos (22\%). However, among the total positive cases, the prevalence of subclinical cases was higher than clinical cases of mastitis. Similarly, quarter based prevalence of mastitis was also higher in subclinical than clinical cases both in buffalo and cattle as shown in the results. These findings are in line with the work of Dhakal et al. [13] who also reported that the mastitis caused by $S$. aureus tends to become subclinical and chronic and has a low response to conventional antibiotic therapy due to its intracellular localization in the mammary gland i.e. epithelial cells. The prevalence of $S$. aureus isolates from mastitic milk samples was more in cattle (90\%) than buffalo (84\%) which indicates more susceptibility of cattle towards $S$. aureus based mastitis than buffalo. The results of isolation, identification and biochemical characterization of indigenous $S$. aureus isolates were also similar to those of Costa et al. [14].

Brain Heart Infusion (BHI) medium was used for the luxuriant production of leukotoxins from indigenous isolates. The same medium was also used by Rainard et al. [14] for the isolation and purification of leukotoxins. The culture supernatants containing crude extracts of leukotoxins were used to determine their toxicity against bovine PMNs. The bovine PMNs are mainly granulocytes $(95 \%)$ particularly neutrophils as also described by Rainard et al. [7]. These are the main phagocytic cells present in milk. The intact monolayer of PMNs in control well without the addition of leukotoxins after 30 minutes of incubation at $37^{\circ} \mathrm{C}$ was found to be round and refractile when observed through inverted microscope. Prévost et al. [15] also had similar findings. They described a remarkable change in the shape of PMNs when come out of blood and enter milk. In milk, PMNs start ingesting milk fat particles and casein which ultimately results in the formation of membrane bounded intracellular vacuoles. This internalization of cell membrane results in the loss of pseudopodia and cell rounding.

Under the influence of leukotoxins, PMNs started to spread and flattened which showed their toxicity towards PMNs. As the concentration of leukotoxins decreased with increasing two fold serial dilutions in TLC assay, the leukotoxicity also decreased and PMNs remained round. The highest dilution which spread more than 95\% PMNs was taken as the end point and it's reciprocal as TLC titer. All these findings are in conformity with the work of Rainard et al. [7]. The results of TLC titers showed that leukotoxins extracted from indigenous $S$. aureus isolates were equally toxic in case of buffalo as well as cattle as similar TLC titers with almost similar GMTs were obtained in both. However there was a significant difference in their leukotoxicity in terms of GMT of TLC titers among subclinical and clinical cases of mastitis. The GMTs were 6 to 8 in subclinical whereas 64 in clinical mastitic cases.

It may be concluded from the present study that the leukotoxins were more toxic to bovine PMNs in clinical 
conditions than subclinical mastitis. However there was no significance difference in terms of their toxicity among buffalo and cattle. It suggests that there may be a major role of these leukotoxins in the virulence of indigenous S. aureus isolates and thus in the pathogenesis of bovine mastitis. Further studies are needed for the purification and molecular characterization of these leukotoxins from indigenous $S$. aureus isolates and to delineate the immunization potential of these toxins to be used as subunit vaccine in future. This may help in the implementation of better disease control programme against bovine mastitis in the country.

\section{CONFLICT OF INTEREST}

The authors confirm that this article content has no conflict of interest.

\section{ACKNOWLEDGEMENTS}

Declared none.

\section{REFERENCES}

[1] Cabral KG, Lämmler C, Zschock M, et al. Pheno and genotyping of Staphylococcus aureus, isolated from bovine milk samples from Sao Paulo State, Brazil. Can J Microbiol 2004; 50: 901-9.

[2] Katsuda K, Hata E, Kobayashi H, et al. Molecular typing of Staphylococcus aureus isolated from bovine mastitic milk on the basis of toxin genes and coagulase gene polymorphisms. Vet Microbiol 2005; 25: 301-5.

[3] Gudding R, McDonald JS, Cheville NF. Pathogenesis of Staphylococcus aureus mastitis: bacteriological, histological and ultra-structural pathologic findings. Am J Vet Res 1984; 45: 2525-31.
[4] Hata E, Katsuda K, Kobayashi H, et al. Bacteriological characteristics of Staphylococcus aureus isolates from humans and bulk milk. J Dairy Sci 2008; 91: 564-9.

[5] Kerr DE, Wellnitz O. Mammary expression of new genes to combat mastitis. J Anim Sci 2002; 81: 38-47.

[6] Peacock SJ, Moore CE, Justice A, et al. Virulent combinations of adhesion and toxin genes in natural populations of Staphylococcus aureus. Infect Immunol 2002; 70: 4987-96

[7] Rainard P, Corrales JC, Barrio MB, et al. Leukotoxic activities of Staphylococcus aureus strains isolated from cows, ewes, and goats with mastitis: importance of LukM/LukF'-PV leukotoxins. Clin Diag Lab Immunol 2003; 10: 272-7.

[8] Haas CJ, Veldkamp KE, Paschal A, et al. Chemotaxis inhibitory protein of Staphylococcus aureus, a bacterial anti-inflammatory agent. J Exp Med 2004; 199: 687-95.

[9] Rainard P. Staphylococcus aureus leucotoxin LukM/F' is secreted and stimulates neutralising antibody response in the course of intramammary infection. Vet Res 2007; 38: 685-96.

[10] Muhammad G. Staphylococci of bovine mammary gland: (I) Conventional and molecular dynamics of infections, (II) Plasmid stability and reproducibility and, (III) interspecific conjugal transfer of antibiotic resistance. Ph.D. Dissertation. Columbus, Ohio, USA: Department of Vet. Prev. Med. The Ohio State Univ 1992.

[11] Carlson GP, Kaneko J. Isolation of leukocytes from bovine peripheral blood. Proc Soc Exp Biol Med 1973; 142: 853-6.

[12] Kozytska S, Staub D, Pawlik MC, et al. Identification of specific genes in Staphylococcus aureus strains associated with bovine mastitis. Vet Microbiol 2010; 145: 360-5.

[13] Dhakal IP, Dhakal P, Koshihara T, et al. Epidemiological and Bacteriological Survey of Buffalo mastitis in Nepal. J Vet Med Sci 2007; 69: 1241-5.

[14] Costa E, Castillo CW, Teixeira CM. Sensitivity of bovine mastitis microorganisms to antibiotics and chemotherapeutic drugs. Pesqui Vet Bras 1985; 5: 65-9.

[15] Prévost G, Mourey L, Colin DA, et al. Staphylococcal poreforming toxins. Curr Top Microbiol Immunol 2001; 257: 53-83. 